\title{
Valuación de un producto estructurado de compra sobre el SX5E cuando la incertidumbre de los rendimientos está modelada con procesos log-estables
}

\author{
Pricing of a structured product on the SX5E when the uncertainty \\ of returns is modeled as a log-stable process
}

\author{
José Antonio Climent Hernández ${ }^{\mathrm{a}, *}$ y Carolina Cruz Matú ${ }^{\mathrm{b}}$ \\ ${ }^{a}$ Universidad Autónoma Metropolitana, México \\ ${ }^{\mathrm{b}}$ Grupo Bolsa Mexicana de Valores, México
}

Recibido el 6 de agosto de 2015; aceptado el 26 de mayo de 2016

Disponible en Internet el 8 de julio de 2017

\section{Resumen}

Se presenta el factor de participación y la valuación de un producto estructurado de primera generación con opciones europeas de compra sobre el Eurostoxx cuando la incertidumbre de los rendimientos está modelada a través de procesos log-estables; así mismo se presentan los estadísticos básicos de los rendimientos del índice, se estiman los parámetros $\alpha$-estables y se compara la valuación de los productos estructurados a través de los modelos log-estable y log-gaussiano utilizando insumos de los mercados de deuda, concluyendo que los inversionistas obtienen rendimientos superiores a los de los mercados de deuda a través de ambos modelos, y que las diferencias de los rendimientos dependen del factor de participación y del valor del índice en la fecha de liquidación.

(C) 2017 Universidad Nacional Autónoma de México, Facultad de Contaduría y Administración. Este es un artículo Open Access bajo la licencia CC BY-NC-ND (http://creativecommons.org/licenses/by-nc-nd/4.0/).

Códigos JEL: G11; G12, G13; D81; C46

Palabras clave: Bonos; Valuación de opciones; Productos estructurados; Distribuciones $\alpha$-estables

\footnotetext{
* Autor para correspondencia.

Correo electrónico: jach@azc.uam.mx (J.A. Climent Hernández).

La revisión por pares es responsabilidad de la Universidad Nacional Autónoma de México.
} 


\begin{abstract}
We report the participation level, we pricing a first generation's European call options on the Eurostoxx structured product, when returns' uncertainty is modeled by log-stable processes, we present the basic statistics of the index's returns, we estimate the $\alpha$-estable parameters, and we compare the structured products pricing by the both log-stable and log-Gaussian models using inputs of the debt markets. We conclude that investors get higher returns than debt markets using both models and returns' differences depend of the participation level and the maturity.

(C) 2017 Universidad Nacional Autónoma de México, Facultad de Contaduría y Administración. This is an open access article under the CC BY-NC-ND license (http://creativecommons.org/licenses/by-nc-nd/4.0/).
\end{abstract}

JEL classification: G11; G12, G13; D81; C46

Keywords: Bonds; Option pricing; Structured products; $\alpha$-Stable distributions

\title{
Introducción
}

Los mercados financieros han evolucionado debido a las tecnologías de la información, a la competencia global, a la ingeniería financiera que ha innovado en el diseño de productos para satisfacer necesidades de cobertura de inversionistas y emisores. Los productos estructurados han tenido éxito debido a que otorgan la posibilidad de inversiones con rendimientos superiores a los instrumentos de deuda cuando las tasas de interés están a la baja. Los productos estructurados son combinaciones de instrumentos de deuda y productos derivados, y son emitidos por instituciones con una calidad crediticia elevada y con un riesgo de crédito calificado como bajo; son atractivos para los inversionistas debido a los rendimientos esperados, la calidad crediticia, la flexibilidad de la estructura de los vencimientos, el diseño de las emisiones, el uso de activos, el acceso a mercados globales que de otra forma serían inaccesibles, y la capacidad para la administración de riesgos de forma eficiente. La regulación de los productos estructurados en México es responsabilidad del Banco de México, la Comisión Nacional Bancaria y de Valores (CNBV) y la Comisión Nacional del Sistema de Ahorro para el Retiro (CONSAR).

McCann y Cilia (1994) analizan los rendimientos de productos estructurados concluyendo que son superiores a los del mercado cuando los rendimientos están a la baja. Lamothe y Pérez (2003) analizan la importancia de la estructuración. Gómez (2007) indica que los productos estructurados permiten a las sociedades de inversión, aseguradoras, bancos y fondos de pensiones obtener rendimientos superiores a los de los mercados de deuda a través de las expectativas y eventualidades de los mercados. Venegas-Martínez (2008) analiza la valuación de las principales notas estructuradas. Castillo (2008) indica el funcionamiento básico de los productos estructurados. Blümke (2009) motiva el uso de productos estructurados como instrumentos de inversión. Mascareñas (2010) muestra que los emisores de productos estructurados, excepcionalmente, adquieren los riesgos implícitos, ya que los transfieren o comparten a través de coberturas utilizando productos derivados, lo que permite diseñar productos que satisfacen las necesidades de inversionistas que están expuestos al riesgo de crédito y que dependen de la calidad crediticia de los emisores. OrtizRamírez, Venegas-Martínez y López-Herrera (2011) indican que la valuación de los productos estructurados tiene una complejidad que necesita entender el comportamiento del mercado, conocer teorías especializadas y disponer de conocimientos sofisticados necesarios para determinar los precios de productos estructurados que son complejos en su diseño (Mascareñas, 1995 y 2010). 
Wallmeier (2011) indica que existe preocupación porque la complejidad y la diversidad de los productos estructurados están acompañadas por una baja transparencia. En la práctica, la información proporcionada a los inversionistas está centrada en los diagramas de pago, por lo que es importante mejorar la información y la comprensión de los inversionistas; los productos estructurados son emitidos por bancos, son negociados en una bolsa organizada o con el banco emisor que cita precios de oferta, de demanda y durante la vigencia del producto; los emisores diseñan productos estructurados atractivos para los inversionistas con perfiles de riesgo-rendimiento que no son fáciles de replicar por instrumentos financieros tradicionales y que proporcionan acceso a clases de activos a los que no tienen acceso directo; los inversionistas tienen dificultades para entender las características de productos complejos, y la valuación de precios justos no siempre está a disposición del público; los estudios empíricos sobre la valuación de productos estructurados en el mercado primario encuentran primas del 2 al $6 \%$ más elevadas que los valores teóricos, los sobreprecios son más pronunciados en mercados emergentes y están relacionados positivamente con la complejidad de los productos; la información padece porque está centrada en el perfil de pagos y no en la probabilidad del perfil de pagos; las preferencias de los inversionistas están caracterizadas por los momentos de la distribución de los rendimientos: rendimiento esperado, volatilidad, asimetría y curtosis; la distribución de los rendimientos para productos estructurados de capital garantizado propone volatilidad baja, asimetría positiva y curtosis media; concluye que los productos estructurados deben ser analizados como un portafolio diversificado, y el riesgorendimiento se debe basar en un análisis de equilibrio de mercado porque las características de asimetría y curtosis de la distribución de los rendimientos se pueden incluir en las primas de riesgo.

Cao y Rieger (2013) demuestran que teóricamente el rendimiento esperado de los productos estructurados es ilimitado, mientras que la restricción para estimar la pérdida máxima esperada a través del valor en riesgo puede ser satisfecha; indican que los productos estructurados son una clase de productos financieros que combinan instrumentos financieros como acciones y derivados para lograr propósitos específicos de inversión; los productos estructurados de capital garantizado permiten a los inversionistas participar de los beneficios potenciales del precio subyacente, mientras están protegidos contra pérdidas potenciales, estos productos se pueden construir combinando una opción de compra y una inversión de interés fijo; desde la crisis financiera de 2008 y el incumplimiento de pagos de productos estructurados, la comprensión del riesgo es un factor crucial y para los inversionistas es una tarea complicada porque el conocimiento de la estructura de pagos es deficiente, y el conocimiento de la estimación de los riesgos es más deficiente; para atraer inversionistas, los emisores diseñan productos estructurados que maximizan el rendimiento esperado y satisfacen la restricción del valor en riesgo, por lo que pueden alcanzar cualquier perfil de rendimiento; definen una medida de riesgo-rendimiento que depende de las ganancias, de las pérdidas y de los parámetros del mercado de tal manera que el riesgo depende solo de las pérdidas del producto, es estrictamente monótona respecto a las pérdidas, es estrictamente monótona respecto a las ganancias y para un nivel de riesgo, el rendimiento está limitado, entonces no permite que los inversionistas solo busquen un rendimiento con un nivel de riesgo y sean engañados; aplican la medida en productos estructurados de capital garantizado, y los perfiles de riesgo y rendimiento cambian cuando se cambia el nivel de garantía: a menor nivel de capital garantizado se obtiene un nivel de riesgo mayor y una medida de rendimiento mayor; concluyen que el riesgo puede ser «barrido debajo de la alfombra» y se pueden diseñar productos estructurados con elevados rendimientos esperados, y los inversionistas que siguen una estrategia para maximizar los rendimientos con un nivel riesgo dado son engañados para tomar riesgos elevados. 
Climent-Hernández y Venegas-Martínez (2013) presentan una gama de distribuciones $\alpha$ estables para modelar series financieras y económicas; analizan el modelo log-estable ortogonal para la valuación de opciones europeas; estiman los parámetros de la distribución de los rendimientos del tipo de cambio peso-dólar a través de los métodos de máxima verosimilitud, tabulación por cuantiles de las distribuciones $\alpha$-estables y regresión sobre la función característica de la muestra; realizan un análisis cualitativo para mostrar la calidad del ajuste de la distribución y un análisis cuantitativo para elegir el mejor ajuste de la distribución; comparan el modelo log-estable con el modelo log-gaussiano y un vector de precios del MexDer, y muestran que el modelo log-estable presenta ventajas sobre el modelo log-gaussiano en la cuantificación de factores de riesgo como curtosis y asimetría, modelando adecuadamente la dinámica de los rendimientos que presentan cúmulos de volatilidad elevada y eventos extremos que tienen un impacto financiero y económico de cuantías superiores a lo que indica la distribución log-gaussiana; presentan un análisis comparativo valuando opciones europeas a través de los modelos log-estable y log-gaussiano que muestra las diferencias en los precios de los pagos contingentes, pero las diferencias entre las valuaciones log-estables y log-gaussianas son inversas debido a un error técnico en las ecuaciones (27) y (28), por lo cual la valuación log-estable es inferior para la valuación de opciones en un intervalo cercano en dinero y superior cuando la valuación está fuera de dinero.

Aguilar-Juárez y Venegas-Martínez (2014) desarrollan una estrategia de inversión con un portafolio de notas estructuradas que garantizan un flujo de efectivo pronosticable al vencimiento y una nota estructurada que inicia su vigencia al vencimiento del portafolio, ofreciendo la posibilidad de obtener un rendimiento mayor al del mercado con un nivel de riesgo bajo; indican que en los mercados de notas estructuradas los inversionistas son adversos al riesgo y aprovechan oportunidades con menor riesgo y mayor rendimiento con capital inicial garantizado o al menos con una parte proporcional; definen un producto estructurado como un portafolio con un instrumento de renta fija o variable y productos derivados que en algunos casos son opciones, y que los más utilizados y negociados son las notas estructuradas con instrumentos de deuda y productos derivados donde las opciones son europeas, estándar o exóticas; concluyen que la administración de riesgo es cada vez más complicada y requiere estrategias que permitan a los inversionistas satisfacer sus necesidades de garantía sobre la inversión y mayor rendimiento; para reducir el riesgo de una inversión, es posible desarrollar estrategias combinando, en un solo producto, productos financieros tradicionales y productos derivados.

Hens y Rieger (2014) demuestran que para inversionistas racionales con creencias correctas y constante de aversión al riesgo, las ganancias de los productos estructurados sobre un portafolio con un activo libre de riesgo y el portafolio de mercado son menores que las tasas del mercado y el resultado es igual si los inversionistas operan continuamente el activo libre de riesgo y el portafolio de mercado sin costo o si compran los activos y los mantienen hasta el vencimiento del producto estructurado; considerando la teoría prospectiva, o inversionistas con creencias erróneas, las ganancias de los pagos pueden ser considerables; indican que los productos estructurados incluyen derivados financieros y el pago al vencimiento depende de uno o más activos; estos productos son emitidos por bancos y están dirigidos a inversionistas minoristas; calculan el producto estructurado óptimo y evalúan si existe y un aumento significativo en la utilidad; concluyen que el aumento de la utilidad de los mejores productos estructurados es de 10 puntos base, y es pequeño comparado con los 200 puntos base pagados por productos estructurados sobre un activo libre de riesgo y el portafolio de mercado sin considerar una compensación por el riesgo contraparte que el inversionista tiene; no existe evidencia de que el modelo de utilidad esperada de NeumannMorgenstern pueda explicar la demanda, pero las utilidades de la teoría prospectiva con creencias no racionales pueden ser superiores. 
Ilin, Koposov y Levina (2014) proponen utilizar los productos estructurados como una estrategia de administración del portafolio de inversión combinando opciones, activos, bonos y swaps; desarrollan un modelo que permite bosquejar la estrategia para construir y administrar el portafolio con un nivel de riesgo regulado; indican que los productos estructurados ofrecen exposición a mercados con características y estructuras diferentes que permiten a los inversionistas precisión para estrategias de inversión específicas; pueden combinar características del mercado de capitales (posibilidad de ganancias ilimitadas), mercado de deuda (renta fija) o depósitos bancarios (límite de riesgo); permiten fijar el riesgo, y el rendimiento es menor que el de la inversión en el subyacente; los compradores son inversionistas privados y fondos de inversión; el mercado de productos estructurados aumenta durante el crecimiento de la volatilidad; los productos estructurados están encaminados para maximizar el valor de los activos de los inversionistas, los beneficios están compuestos por los rendimientos de los activos libres de riesgo y los rendimientos de las opciones, y si las opciones no se ejercen, el valor de los activos se reduce; la tasa de interés libre de riesgo debe ser el límite mínimo del rendimiento; las desventajas son: primero, la estrategia de inversión no se puede planear totalmente, es decir, existe el riesgo de tomar decisiones equivocadas, segundo, una parte significativa de los activos se debe invertir en instrumentos libres de riesgo, con rentabilidades bajas; son un acercamiento progresivo a la administración del capital; concluyen que los emisores aprovechan las oportunidades de campañas de promoción para ofrecer inversiones inusuales.

Schroff, Meyer y Burghof (2015) estudian el impacto de la demanda de información de los inversionistas sobre la negociación de inversión emitida por bancos utilizando productos estructurados que están diseñados para inversionistas minoristas; indican que la demanda de información predice positivamente la actividad de negociación especulativa; encuentran una relación positiva entre la demanda de información del mercado y la especulación al invertir; la demanda de información no estimula a los inversionistas a tomar posturas largas o cortas, lo que manifiesta una baja eficiencia; indican que la hipótesis de que los mercados financieros son informativamente eficientes descansa sobre el supuesto de que los precios responden de inmediato a la información, sin embargo, la incorporación de la información en los precios requiere que los inversionistas tengan acceso a toda la información y que pongan atención a la información para que esta esté considerada en la toma de decisiones; cuando los inversionistas minoristas están interesados en invertir en una empresa, es probable que obtengan la información a través de motores de búsqueda y los inversionistas institucionales son más propensos a utilizar fuentes de información de proveedores de datos financieros como Bloomberg; el mercado de productos estructurados se puede dividir en inversiones de largo plazo con características de pagos conservadoras e inversiones de apalancamiento especulativo diseñadas para estrategias de corto plazo; el diseño permite a los inversionistas obtener beneficios por aumento o caída de los precios subyacentes; los productos estructurados de inversión son para estrategias de inversión a largo plazo y son utilizados por inversionistas minoristas en planes de ahorro; concluyen que existe un efecto positivo en la demanda de información en la actividad de negociación especulativa, no existe relación en la actividad de inversión a largo plazo, y por lo tanto la eficiencia de información del mercado de productos estructurados es limitada.

El objetivo es valuar un producto estructurado que incluye un bono y una opción europea de compra sobre el Eurostoxx, por lo que se propone utilizar la teoría de valuación de opciones y, dada la evidencia de que los rendimientos presentan leptocurtosis y asimetría, se propone que la valuación de las opciones se realice a través del modelo propuesto en la investigación de ClimentHernández y Venegas-Martínez (2013), innovando en la valuación de productos estructurados de capital garantizado de primera generación utilizando distribuciones log-estables para modelar 
adecuadamente la dinámica de los rendimientos (leptocurtosis, asimetría, fluctuaciones lejanas a la moda o valores extremos, propiedad de estabilidad o persistencia) con cúmulos de volatilidad elevada y considerando eventos extremos poco probables en el contexto de la distribución gaussiana que realmente tienen frecuencias superiores y un impacto financiero y económico de cuantías superiores en los mercados, lo que permite comparar la valuación de los productos estructurados a través del modelo log-estable y log-gaussiano, que es el caso límite del modelo log-estable cuando $\alpha=2$, por lo que las aplicaciones log-estables son más amplias que la aplicación log-gaussiana.

El trabajo está organizado de la forma siguiente: en la siguiente sección se desarrolla el producto estructurado de capital garantizado referenciado al índice SX5E, el cálculo del capital que se invierte en el bono y el cálculo del factor de participación para opciones de compra sobre el índice; en la tercera sección se muestra la valuación del bono utilizando una tasa de interés instantánea equivalente a la tasa de interés simple y la valuación de las opciones log-gaussianas y log-estables; en la cuarta sección se presenta el análisis de los rendimientos del índice, la estimación de los estadísticos básicos, la estimación de los parámetros $\alpha$-estables, las pruebas de bondad de ajuste, el desempeño del índice y el desempeño de las volatilidades; en la quinta sección se presenta la estimación del parámetro de escala implícito, el desempeño de las volatilidades y escalas históricas, el desempeño de las opciones log-gaussianas y log-estables, el desempeño del bono, el cálculo para la monetización de las opciones y el desempeño de los productos estructurados log-gaussianos y log-estables; en la sexta sección se presentan las conclusiones del trabajo de investigación, y, por último, la bibliografía.

\section{El producto estructurado de compra}

El producto estructurado objeto de estudio es un portafolio compuesto por un bono y la posición larga de opciones europeas de compra sobre el índice SX5E emitidas el día 4 de septiembre de 2014, con vencimiento el 28 de agosto de 2017 (fixing date) y con fecha de liquidación el 31 de agosto de 2017 (plazo de vigencia de 1,092 días para la maduración del bono). El producto estructurado de capital garantizado es conocido como bono bancario estructurado de compra sin pérdida de capital al vencimiento y referenciado al Eurostoxx, y tiene un porcentaje retornable del monto inicial al vencimiento del $100 \%$, en pesos, cuando se presenta una baja en el índice SX5E, es decir, tiene una pérdida máxima del 0\%. El producto estructurado debe invertir en el bono la cantidad siguiente:

$$
B_{0}=B_{T} \exp (-i T)
$$

donde $B_{T}$ es el valor nominal del bono, $T$ es el tiempo de maduración del bono, $\tau=T-t$ es el tiempo remanente del bono e $i$ es la tasa de interés instantánea equivalente a la tasa de interés simple $i_{s}$, de tal forma que $i=\frac{365}{\tau} \ln \left(1+i_{s} \frac{\tau}{360}\right)$ es la tasa aplicada en la estructuración. El capital restante para la posición larga en opciones europeas de compra sobre el Eurostoxx es:

$$
C_{0}=B_{T}-B_{0}
$$

Entonces el factor de participación se obtiene a través del cociente:

$$
F_{0}=\frac{C_{0}}{c\left(0, M_{0}\right)}
$$

donde $c\left(0, M_{0}\right)$ es el valor de opción europea de compra sobre el Eurostoxx en la fecha de emisión. 
La nota estructurada de compra (call note) de capital garantizado protege el valor nominal de la inversión al ciento por ciento y el rendimiento futuro es el que se encuentra en riesgo, ya que el peor escenario tiene como valor de liquidación (payoff) de la opción un valor nulo, mientras el valor de liquidación del bono es $B_{T}$. Entonces el inversionista recibe cuando menos el capital invertido más los intereses, si el Eurostoxx incrementa su valor; el pago de la opción incrementa el valor de la nota estructurada de compra. La nota estructurada $c\left(t, M_{t}, B_{t}\right)$ sobre una opción emitida sobre el Eurostoxx es un instrumento de deuda con rendimiento variable implícito (cupón cero) equivalente al valor de mercado del bono (renta fija) que se liquida en pesos y en una sola exhibición al vencimiento del producto estructurado más el valor del rendimiento (renta variable) del factor de participación de la opción $c\left(t, M_{t}\right)$ que también se liquida en pesos y en una sola exhibición al vencimiento del producto estructurado donde los objetivos de los inversionistas son especular aprovechando las expectativas a la alza del Eurostoxx, obtener una cobertura para riesgos existentes en el portafolio de inversión u obtener una inversión en un mercado al que no tienen acceso directamente. La nota estructurada de compra sobre el Eurostoxx es de primera generación, ya que las opciones no son exóticas; solo hay una tasa de rendimiento, la fecha de maduración del bono coincide con la fecha de liquidación de las opciones y la tasa de rendimiento es la tasa LIBOR-EURO, porque las opciones sobre el índice son emitidas por una institución europea y el inversionista es el emisor que monetiza en pesos las ganancias por las opciones. Este producto estructurado tiene éxito debido a la comprensión para inversionistas y emisores, donde los inversionistas esperan que el valor del índice incremente y los emisores deben transferir el riesgo de precio a través de productos derivados. Durante septiembre de 2014 el monto en pesos de las notas estructuradas de compra ascendió al 35.3465\% del monto negociado, ocupando el primer lugar.

\section{Valuación del producto estructurado de compra}

La nota estructurada de compra sobre el Eurostoxx es un portafolio compuesto por un bono y el factor de participación en opciones europeas de compra sobre el Eurostoxx, entonces es necesario valuar el bono y las opciones con insumos del mercado. Los proveedores de precios encargados de dar certidumbre al mercado calculan el valor teórico de las opciones europeas sobre el índice, el precio teórico del bono y publican el valor teórico del producto estructurado. La evidencia de que los rendimientos subyacentes presentan leptocurtosis y asimetría, como se ha observado en los trabajos de Dostoglou y Rachev (1999), Č́ízek, Härdle y Weron (2005) y Climent-Hernández y Venegas-Martínez (2013), lleva a proponer que la valuación de las opciones sea realizada a través del modelo propuesto en el trabajo de investigación de Climent-Hernández y Venegas-Martínez (2013) utilizando distribuciones log-estables que modelan adecuadamente la leptocurtosis, la asimetría, las fluctuaciones lejanas a la moda (valores extremos) y la propiedad de estabilidad (persistencia) de los rendimientos porque son una alternativa efectiva para modelar series financieras y económicas con cúmulos de volatilidad elevada, valores extremos con frecuencias superiores a la distribución log-gaussiana y que tienen un impacto financiero y económico de cuantías mayores, además de que satisfacen el teorema del límite central debido a que los rendimientos se encuentran en el dominio de atracción de una ley log-estable donde la distribución log-gaussiana es el caso límite del modelo log-estable cuando $\alpha=2$ y se ha mostrado que no es eficiente para modelar adecuadamente la leptocurtosis, la asimetría, los eventos lejanos al parámetro de localización y la propiedad de estabilidad observada en los rendimientos de las series financieras y económicas, mientras que los rendimientos log-estables satisfacen la propiedad de estabilidad que permite modelar las series empíricas optimando el desempeño del sistema, 
por lo que las aplicaciones de las distribuciones log-estables son más amplias que las aplicaciones de la distribución log-gaussiana, que considera eventos extremos y de alto impacto como poco probables y que son más frecuentes para las distribuciones $\alpha$-estables, lo que permite comparar la valuación de los productos estructurados a través de los modelos log-estable y log-gaussiano. La valuación del bono se realiza con la ecuación siguiente:

$$
B_{t}=B_{T} \exp (-i \tau)
$$

donde el instante $t \in[0, T]$. La valuación de las opciones europeas de compra se realiza a través del modelo log-gaussiano:

$$
c\left(t, M_{t}\right)=M_{t} \exp (-r \tau) \Phi\left(d_{1}\right)-S \exp (-i \tau) \Phi\left(d_{2}\right)
$$

donde:

$$
d_{1}=\frac{\ln \left(\frac{M_{t}}{S}\right)+\left(i-r+\frac{\sigma^{2}}{2}\right) \tau}{\sigma \sqrt{\tau}} \quad \text { y } \quad d_{2}=d_{1}-\sigma \sqrt{\tau}
$$

utilizando el supuesto de que los rendimientos tienen una distribución log-gaussiana donde $M_{t}$ es valor del índice, $S$ es el precio de liquidación (3,172.63 puntos), $r$ es la tasa de dividendos del índice y $\sigma$ es la volatilidad de los rendimientos del índice. La valuación de las opciones de compra se realiza con el modelo log-estable:

$$
c\left(t, M_{t}\right)=\left\{\begin{array}{l}
M_{t} \exp (-r \tau) \Phi(-d ; \alpha, \beta)-S \exp (-i \tau) \Phi(d ; \alpha,-\beta) \\
M_{t} \exp (-r \tau) \Phi(d ; \alpha,-\beta)-S \exp (-i \tau) \Phi(-d ; \alpha, \beta)
\end{array}\right.
$$

donde:

$$
d=\frac{\ln \left(\frac{M_{t}}{S}\right)+\left(i-r-\beta \gamma^{\alpha} \sec (\theta)\right) \tau}{\frac{1}{\alpha}} \text { y } \quad \theta=\frac{\alpha \pi}{2}
$$

utilizando el modelo propuesto por Climent-Hernández y Venegas-Martínez (2013), donde $\beta$ es el parámetro de asimetría, $\gamma$ es el parámetro de escala y $\alpha$ es el parámetro de estabilidad de las distribuciones $\alpha$-estables.

\section{Análisis de los rendimientos del Eurostoxx}

El subyacente utilizado en esta investigación es el SX5E, que es un índice bursátil compuesto por las 50 acciones más líquidas de la zona euro. El desempeño del Eurostoxx durante el periodo del 4 de enero de 2010 al 3 de septiembre de 2014 se presenta en la figura 1, donde se puede observar el desempeño del Eurostoxx hasta el día hábil inmediato anterior de la emisión del bono bancario estructurado de compra sin pérdida de capital al vencimiento y referenciados al Eurostoxx con 1,201 observaciones que presentan un mínimo de 1,995.01 y un máximo de 3,314.80 puntos.

\section{Estimación de los estadísticos básicos de los rendimientos del Eurostoxx}

El periodo utilizado para estimar los parámetros $\alpha$-estables de la distribución de los rendimientos del Eurostoxx es del día 4 de enero de 2010 al 3 de septiembre de 2014, periodo que 


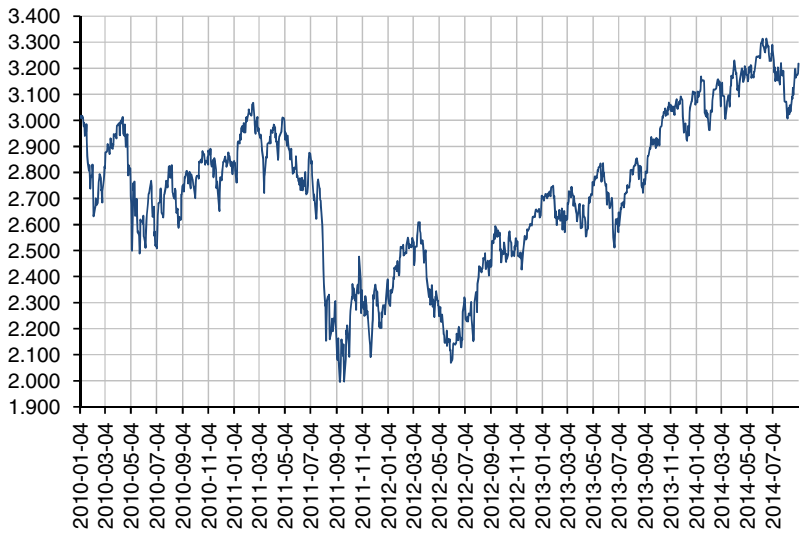

Figura 1. Desempeño del Eurostoxx.

Fuente: elaboración propia a través de hoja de cálculo.

comprende 1,201 observaciones para el índice y 1,200 observaciones para los rendimientos logarítmicos. Los rendimientos diarios del Eurostoxx se presentan en la figura 2, donde se puede observar el desempeño de los rendimientos diarios del Eurostoxx que presentan un mínimo de $-6.3182 \%$ y un máximo de $9.8466 \%$. El desempeño de las volatilidades históricas anuales del Eurostoxx se presenta en la figura 3, donde se puede observar el desempeño de las volatilidades históricas anuales que presentan un mínimo de $1.08 \%$ y un máximo de $34.51 \%$. Las volatilidades históricas anuales presentan una tendencia a la baja desde el 11 de diciembre de 2011 hasta el día hábil inmediato anterior a la emisión del producto estructurado. La estimación de los estadísticos básicos de los rendimientos diarios del Eurostoxx se presenta en la tabla 1, donde el promedio indica que los rendimientos del Eurostoxx se aprecian. El coeficiente asimetría positivo indica que los rendimientos tienen una distribución que se extiende hacia valores positivos con mayor frecuencia que hacia valores negativos. El coeficiente de curtosis indica que la distribución de los rendimientos es leptocúrtica con respecto a la distribución gaussiana, por lo que los rendimientos del Eurostoxx tienen una distribución asimétrica y leptocúrtica.

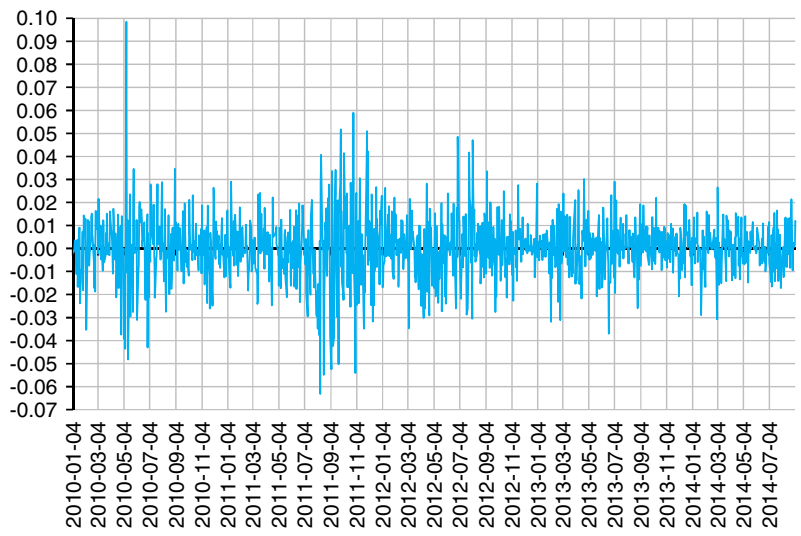

Figura 2. Desempeño de los rendimientos del Eurostoxx.

Fuente: elaboración propia a través de hoja de cálculo. 


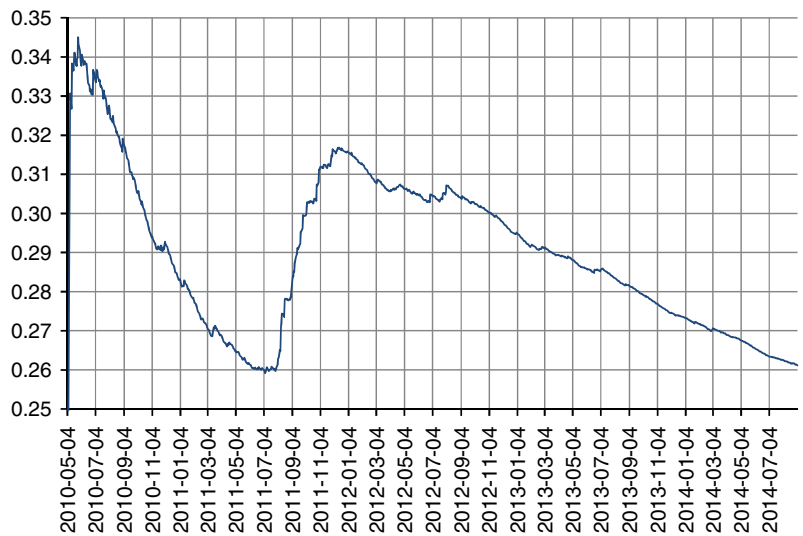

Figura 3. Desempeño de las volatilidades históricas anuales del Eurostoxx.

Fuente: elaboración propia a través de hoja de cálculo.

Tabla 1

Estimación de los estadísticos básicos de los rendimientos del Eurostoxx

\begin{tabular}{lllllll}
\hline Subyacente & Mínimo & Máximo & Promedio & Desviación & Asimetría & Curtosis \\
\hline Eurostoxx & -0.063182 & 0.098466 & 0.000054 & 0.013671 & 0.088444 & 4.055143 \\
\hline
\end{tabular}

Fuente: elaboración propia con datos de MexDer a través de hoja de cálculo.

\section{Estimación de los parámetros $\alpha$-estables de los rendimientos del Eurostoxx}

Las características de los rendimientos del Eurostoxx indican que la distribución es asimétrica y leptocúrtica; para modelar los rendimientos se realiza la estimación de parámetros $\alpha$-estables a través del método de estimación de verosimilitud máxima, que son los valores que maximizan la función de verosimilitud $\ell\left(\alpha, \beta, \gamma, \delta \mid X_{1}, \ldots, X_{n}\right)=\sum_{k=1}^{n} \log \left(X_{k} \mid \alpha, \beta, \gamma, \delta\right)$ y que es el estimador asintóticamente más eficiente, y que está dado por la matriz de información de Fisher, y la función de densidad puede ser expresada en términos de funciones $\mathrm{G}$ de Meijer, representación que no es práctica para evaluar funciones de densidad $\alpha$-estables de acuerdo con la investigación de Nolan (2001). La estimación de parámetros $\alpha$-estables al 95\% de confianza se presenta en la tabla 2.

Los parámetros de estabilidad y asimetría de los rendimientos del Eutostoxx presentados en la tabla 2 son consistentes con los resultados de las investigaciones de Dostoglou y Rachev (1999), Č́ížek et al. (2005), Scalas y Kim (2006), Contreras y Venegas-Martínez (2011) y ClimentHernandez y Venegas-Martínez (2013). El parámetro de estabilidad con un intervalo de confianza del $95 \%$ se encuentra entre los valores $1.6087 \leq \alpha \leq 1.7803$ e indica que la distribución de los rendimientos es leptocúrtica; el parámetro de asimetría con un intervalo de confianza del $95 \%$ se

Tabla 2

Estimación de parámetros $\alpha$-estables de los rendimientos del Eurostoxx

\begin{tabular}{lllll}
\hline Subyacente & $\alpha$ & $\beta$ & $\gamma$ & $\delta$ \\
\hline Eurostoxx & $1.6945 \pm 0.0858$ & $0.1707 \pm 0.2349$ & $0.007851 \pm 0.000427312$ & $0.000138 \pm 0.000784006$
\end{tabular}

Fuente: elaboración propia con datos de MexDer y el programa STABLE.EXE. 
Tabla 3

Resultados de la prueba Kolgomorov-Smirnov

\begin{tabular}{llllll}
\hline Distribución & $D$ & $D_{0.90}$ & $D_{0.95}$ & $D_{0.99}$ & Resultado \\
\hline Gaussiana & 0.06187 & 0.02210 & 0.02415 & 0.02792 & Rechazar $H_{0}$ \\
$\alpha$-estable & 0.03066 & 0.03292 & 0.03653 & 0.04379 & No rechazar $H_{0}$ \\
\hline
\end{tabular}

Fuente: elaboración propia a través de hoja de cálculo.

encuentra entre los valores $-0.4056 \leq \beta \leq 0.0642$ e indica que la distribución se extiende hacia el extremo izquierdo con mayor frecuencia que hacia el extremo derecho. Los resultados anteriores indican que los rendimientos del Eurostoxx presentan leptocurtosis y asimetría negativa.

\section{Prueba de bondad de ajuste Kolmogorov-Smirnov}

El análisis cuantitativo para probar la hipótesis nula $\mathrm{H}_{0}$ de que los rendimientos del Eurostoxx presentan una distribución $\alpha$-estable contra la hipótesis alternativa $\mathrm{H}_{1}$ de que los rendimientos no presentan una distribución $\alpha$-estable se realiza a través del estadístico Kolmogorov-Smirnov, que se presenta en la tabla 3 .

A partir de la tabla 3 y con niveles de significación del 10, del 5 y del 1\%, se concluye que se debe rechazar la hipótesis nula de que los rendimientos del Eurostoxx presentan una distribución gaussiana y que no se debe rechazar la hipótesis nula de que los rendimientos del Eurostoxx presentan una distribución $\alpha$-estable.

\section{Prueba de bondad de ajuste Anderson-Darling}

Otra prueba para la hipótesis nula $\mathrm{H}_{0}$ de que los rendimientos presentan una distribución $\alpha$ estable contra la hipótesis alternativa $\mathrm{H}_{1}$ de que los rendimientos no presentan una distribución $\alpha$-estable se realiza a través del estadístico de bondad de ajuste Anderson-Darling, que se presenta en la tabla 4.

De los resultados de la tabla 4 y con niveles de significación del 10, del 5 y del 1\%, se concluye que se debe rechazar la hipótesis nula de que los rendimientos del Eurostoxx presentan una distribución gaussiana y que no se debe rechazar la hipótesis nula de que los rendimientos del Eurostoxx presentan una distribución $\alpha$-estable estándar $f_{X}(x, 1.6945,-0.1707)$ en un espacio de probabilidad fraccionario. La distribución log-gaussiana y las distribuciones log-estables con $\alpha=1.6945$ y los tres parámetros de asimetría se presentan en la figura 4 , donde se puede observar la distribución log-gaussiana estándar $f_{X}(x, 2)$, con la línea cielo; la distribución log-estable estándar $f_{X}(x, 1.694,-0.406)$, con línea discontinua roja, presenta asimetría negativa, indicando que la moda está ubicada a la derecha de la media, moda y mediana de la distribución log-gaussiana; la distribución log-estable estándar $f_{X}(x, 1.694,-0.171)$, con línea marino, presenta asimetría negativa, indicando que la moda está ubicada a la derecha de la media, moda y mediana de la distribución log-gaussiana; la distribución log-estable estándar $f_{X}(x, 1.694,0.064)$, con línea punteada

Tabla 4

Resultados de la prueba Anderson-Darling

\begin{tabular}{llllll}
\hline Distribución & \multicolumn{1}{c}{$A^{2}$} & $A_{0.90}^{2}$ & $A_{0.95}^{2}$ & $A_{0.99}^{2}$ & Resultado \\
\hline Gaussiana & 10.27164 & 0.63235 & 0.75241 & 1.03557 & ${\text { Rechazar } \mathrm{H}_{0}}$ \\
$\alpha$-estable & 1.18344 & 1.93406 & 2.49336 & 2.85856 & No rechazar $\mathrm{H}_{0}$ \\
\hline
\end{tabular}

Fuente: elaboración propia a través de hoja de cálculo. 


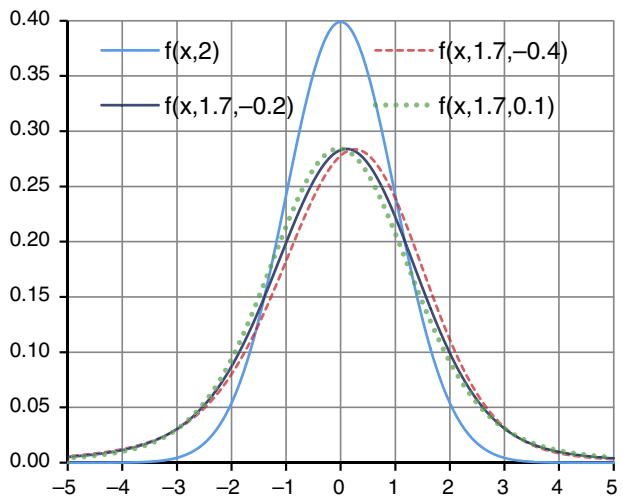

Figura 4. Distribución log-gaussiana y distribuciones log-estables.

Fuente: elaboración propia a través de hoja de cálculo.

verde, presenta asimetría positiva, indicando que la moda está ubicada a la izquierda de la media, moda y mediana de la distribución log-gaussiana (origen). La distribución log-gaussiana indica que eventos cercanos al origen ocurren con mayor frecuencia de lo que realmente ocurre con los rendimientos del Eurostoxx, y también indica que los eventos lejanos al origen (valores extremos) ocurren con menor frecuencia de lo que realmente ocurre con los rendimientos del Eurostoxx, por lo que se puede observar que la distribución log-gaussiana sobrestima eventos de bajo impacto financiero y económico cuando $-1.53<x<1.03$ y subestima eventos de alto impacto financiero $\mathrm{y}$ económico como ganancias o pérdidas cuantiosas cuando $x \leq-1.53$ y cuando $x \geq 1.03$, respectivamente, para la distribución $f_{X}(x, 1.694,-0.406)$; también sobrestima eventos de bajo impacto financiero y económico cuando $-1.41<x<1.17$ y subestima eventos de alto impacto financiero y económico como ganancias o pérdidas cuantiosas cuando $x \leq-1.41$ y cuando $x \geq 1.18$, respectivamente, para la función de distribución $f_{X}(x, 1.694,-0.171)$, y sobrestima eventos de bajo impacto financiero y económico cuando $-1.24<x<1.33$ y subestima eventos de alto impacto financiero y económico como ganancias o pérdidas cuantiosas cuando $x \leq-1.24$ y cuando $x \geq 1.33$, respectivamente, para la función de distribución $f_{X}(x, 1.694,0.064)$. La distribución log-gaussiana y las distribuciones log-estables con los tres parámetros de estabilidad se presentan en la figura 5 , en la que se puede observar que la distribución log-gaussiana también sobrestima eventos de bajo impacto financiero y económico para las distribuciones $f_{X}(x, 1.609)$, línea discontinua color rojo, $f_{X}(x, 1.694)$, línea marino, y $f_{X}(x, 1.780)$, línea discontinua color verde, y también subestima eventos de alto impacto financiero y económico para las distribuciones $f_{X}(x, 1.609)$, línea discontinua roja, $f_{X}(x, 1.694)$, línea marino, y $f_{X}(x, 1.780)$, línea discontinua verde, donde la menor sobrestimación se presenta en la distribución $f_{X}(x, 1.609,-0.406)$, la mayor sobrestimación se presenta en la distribución $f_{X}(x, 1.780,-0.406)$, la menor subestimación se presenta en $f_{X}(x, 1.780,-0.406)$ y la mayor subestimación se presenta en $f_{X}(x, 1.609,-0.406)$; por lo tanto, se puede concluir que la leptocurtosis y el sesgo de la distribución de los rendimientos del Eurostoxx hacen que los eventos de menor impacto financiero y económico sean sobrestimados por la distribución log-gaussiana y los eventos de mayor impacto financiero y económico sean subestimados por la distribución log-gaussiana, beneficiando a los emisores y perjudicando a los inversionistas cuando el precio de liquidación está en un intervalo cercano al precio del mercado en el momento de negociación. El desempeño del Eurostoxx durante el periodo de vigencia (0409-2014 al 04-05-2015) del producto estructurado se presenta en la figura 6, donde se observa 


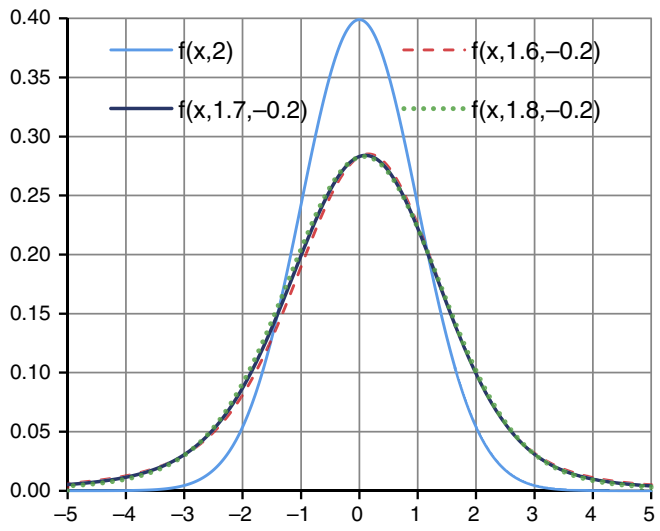

Figura 5. Distribución log-gaussiana y distribuciones log-estables.

Fuente: elaboración propia a través de hoja de cálculo.

el desempeño del Eurostoxx (línea marino) durante el periodo de valuación con 170 observaciones que presentan un mínimo de 2,874.65 y un máximo de 3,828.78 puntos; las ganancias se presentan debido al valor del índice SX5E a partir del 16 de enero de 2015, alcanzando un máximo el 13 de abril de 2015. El producto estructurado de capital garantizado tiene un valor referenciado al Eurostoxx que otorga ganancias a los inversionistas cuando el índice supera el precio de liquidación $S=3,172.63$ puntos (línea discontinua roja) y tiene un valor garantizado que asciende a $\$ 100.00$ (cien pesos 00/100 M.N.). El estado de resultados del producto estructurado presenta un comportamiento parecido a la posición larga de una opción europea de compra sobre el Eurostoxx equivalente a una estrategia en donde los inversionistas especulan sobre el puntaje del índice, esperando que este supere los 3,172.63 puntos en la fecha de vencimiento, lo que genera ganancias para los inversionistas; cuando el Eurostoxx no supera los 3,172.63 puntos en la fecha de vencimiento, los inversionistas reciben el valor nominal del bono bancario por la cantidad de cien pesos. El estado de resultados de la estrategia de compra se presenta en la figura 7, en la que se puede observar que las ganancias del producto estructurado de capital garantizado provienen del valor Eurostoxx, y mientras este supera los 3,172.63 puntos en la fecha de vencimiento de

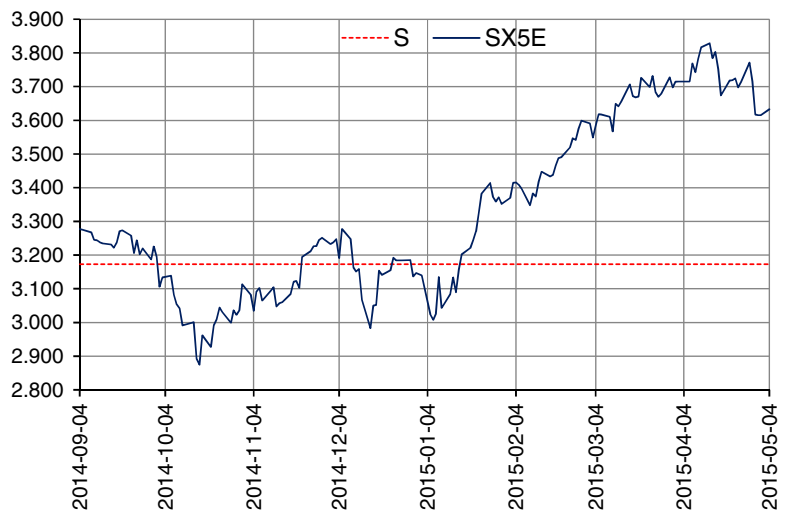

Figura 6. Desempeño del Eurostoxx durante el periodo de valuación.

Fuente: elaboración propia a través de hoja de cálculo. 


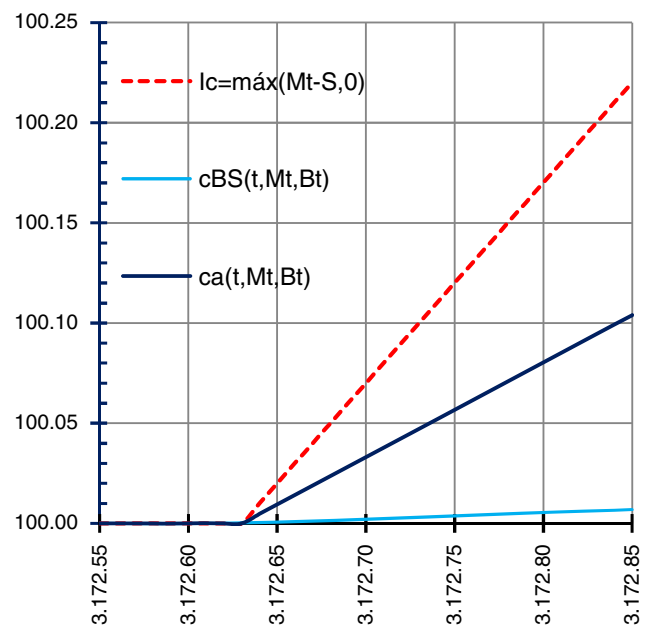

Figura 7. Estado de resultados del producto estructurado en la fecha de liquidación. Fuente: elaboración propia a través de hoja de cálculo.

la opción, las ganancias incrementan en proporción al incremento del índice. La línea discontinua roja representa el valor intrínseco de las opciones de compra, que es el valor máximo de la diferencia del precio de mercado y el precio de liquidación o cero:

$$
I_{c}=\max \left(M_{t}-S, 0\right)
$$

la línea marino representa la ganancia del producto estructurado log-estable considerando el factor de participación $F_{0}$ y la monetización de las opciones que es cien veces el valor máximo del cociente del valor de las opciones sobre el Eurostoxx en la fecha de vencimiento y el precio de liquidación o cero:

$$
\underset{M_{T}}{100 \max }\left(\frac{c\left(T, M_{T}\right)}{S}, 0\right)
$$

donde el valor de las opciones en la fecha de vencimiento es:

$$
c\left(T, M_{T}\right)=\max \left(M_{T}-S, 0\right)
$$

y representa las ganancias adicionales por la inversión en las opciones log-estables y la línea cielo representa la ganancia del producto estructurado log-gaussiano considerando el factor de participación y la monetización de la opción log-gaussiana; como se puede apreciar, las utilidades en la fecha de vencimiento del producto estructurado log-estable son superiores a las log-gaussianas cuando valor del índice supera el precio de liquidación al vencimiento, y esto se debe al factor de participación, que en el caso log-estable incluye en la estructura del portafolio quince opciones contra una opción en el portafolio log-gaussiano, potenciando las ganancias del portafolio logestable por el pago al vencimiento de la opción. La comparación de las volatilidades históricas e implícitas anuales durante el periodo de valuación se presenta en la figura 8, donde se muestra la comparación de las volatilidades históricas (línea cielo) e implícitas (línea marino) anuales durante el periodo de valuación; las volatilidades implícitas presentan un mínimo del 18.8544\% y un máximo del $25.9993 \%$, con un diferencial de 715 puntos porcentuales, y las volatilidades históricas presentan un mínimo del $25.8675 \%$ y un máximo del $26.2418 \%$, con un diferencial 


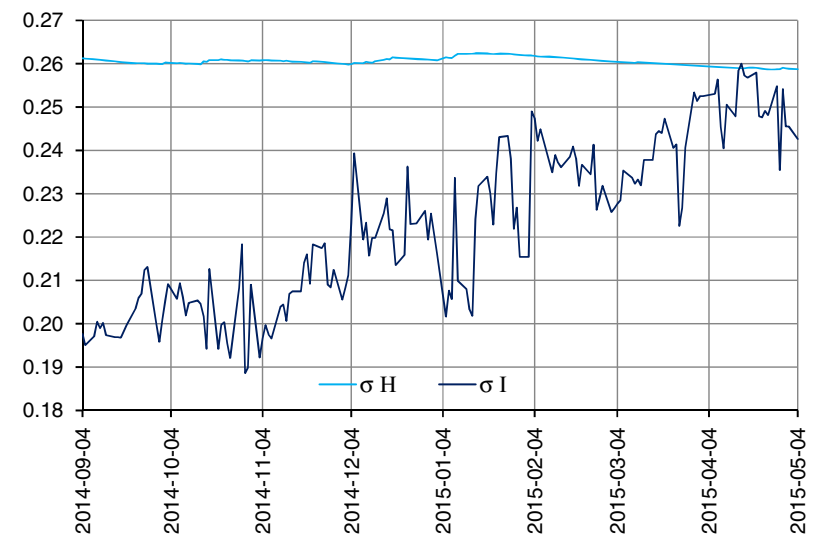

Figura 8. Comparación de las volatilidades históricas e implícitas anuales.

Fuente: elaboración propia a través de hoja de cálculo.

de 38 puntos porcentuales. Se puede observar que el 15 de abril de 2015 es el único día que la volatilidad implícita es mayor que la histórica, lo que significa que las opciones europeas de compra sobre el Eurostoxx están subvaluadas excepto el 15 de abril de 2015, porque se utiliza la volatilidad implícita en la valuación.

\section{Valuación del producto estructurado de compra sobre el Eurostoxx}

La valuación del producto estructurado depende de insumos como el Eurostoxx, la volatilidad implícita de las opciones sobre el Eurostoxx, la tasa de interés LIBOR-EURO, la tasa de interés de los dividendos del índice, el precio de liquidación, el tiempo remanente para la valuación de las opciones europeas de compra a través del modelo log-gaussiano y la tasa cupón cero para la valuación del bono. Para el modelo log-estable no se cuenta con el parámetro de escala implícito, por lo que se propone realizar el cálculo a través de la relación teórica siguiente:

$$
\gamma^{\alpha}=\left\{\begin{array}{cc}
-\frac{\sigma^{\alpha} \cos (\theta)}{\alpha} & \text { si } 0<\alpha<2 \\
\frac{\sigma^{2}}{2} & \text { si } \alpha=2
\end{array}\right.
$$

donde $\sigma$ es la volatilidad implícita anual.

Mediante la ecuación (9) se calculan las escalas implícitas anuales para la valuación de las opciones europeas de compra del modelo log-estable utilizando los mismos factores endógenos: el índice, la tasa de interés LIBOR-EURO, la tasa de dividendos del índice, los mismos factores exógenos: el precio de liquidación y el tiempo remanente; y también la misma tasa cupón cero para la valuación del bono. La comparación de las escalas anuales durante el periodo de valuación se presenta en la figura 9, donde se muestra la comparación de las volatilidades históricas (línea discontinua cielo), las volatilidades implícitas (línea marino), las escalas implícitas (línea roja) y las escalas históricas (línea punteada púrpura). Las escalas implícitas presentan un mínimo del $12.8685 \%$ y un máximo del $17.7450 \%$, con un diferencial de 488 puntos porcentuales, y las escalas históricas presentan un mínimo del $25.1359 \%$ y un máximo del $26.0497 \%$, con un diferencial de 


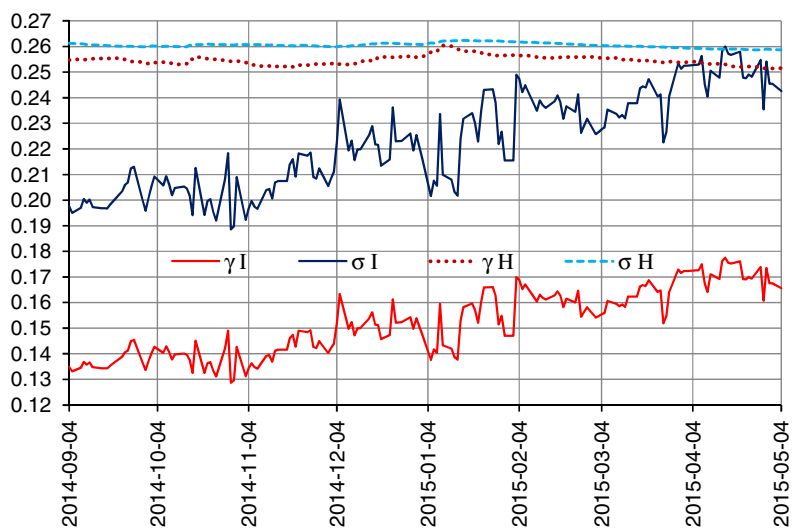

Figura 9. Comparación de las volatilidades y escalas históricas e implícitas anuales. Fuente: elaboración propia a través de hoja de cálculo.

92 puntos porcentuales. Se puede observar que las escalas implícitas siempre son menores que las escalas históricas. Los límites para la valuación de opciones europeas son:

$$
\max \left(M_{t} \exp (-r \tau)-S \exp (-i \tau), 0\right) \leq c\left(t, M_{t}\right) \leq M_{t}
$$

Los precios de las opciones de compra deben ser positivos y deben ser mayores que la diferencia entre el valor presente del precio subyacente a la tasa de dividendos del índice y el valor presente del precio de liquidación a la tasa doméstica, y deben ser menores que el precio del subyacente para evitar oportunidades de arbitraje. La valuación de las opciones europeas de compra a través de los modelos log-gaussiano y log-estable utilizando se presenta en la figura 10, en la que se muestran las valuaciones de las opciones europeas de compra a través del modelo log-gaussiano (línea cielo), las valuaciones de las opciones europeas de compra a través del modelo log-estable (línea marino) y los límites inferiores para las valuaciones de las opciones europeas de compra (línea discontinua roja); las valuaciones log-gaussianas presentan un mínimo de $\$ 154.5342$ y un máximo de $\$ 736.8478$, con un diferencial de $\$ 582.3136$, mientras que las valuaciones log-estables

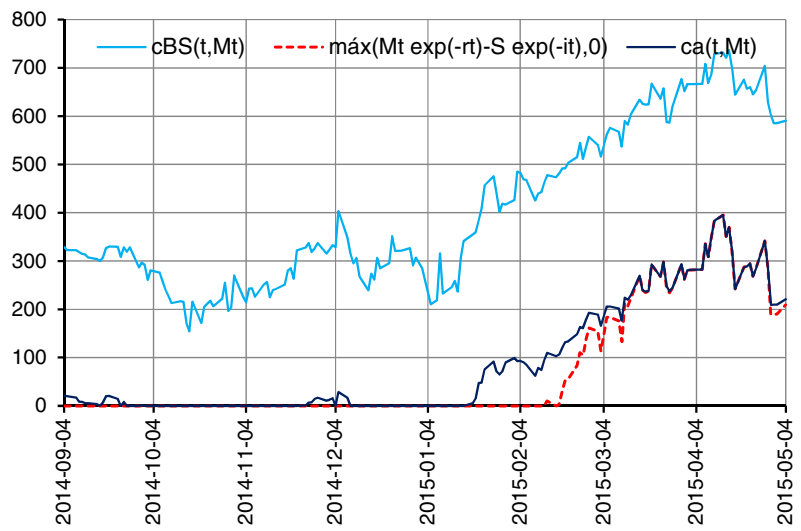

Figura 10. Valuación de opciones europeas de compra. Fuente: elaboración propia a través de hoja de cálculo. 


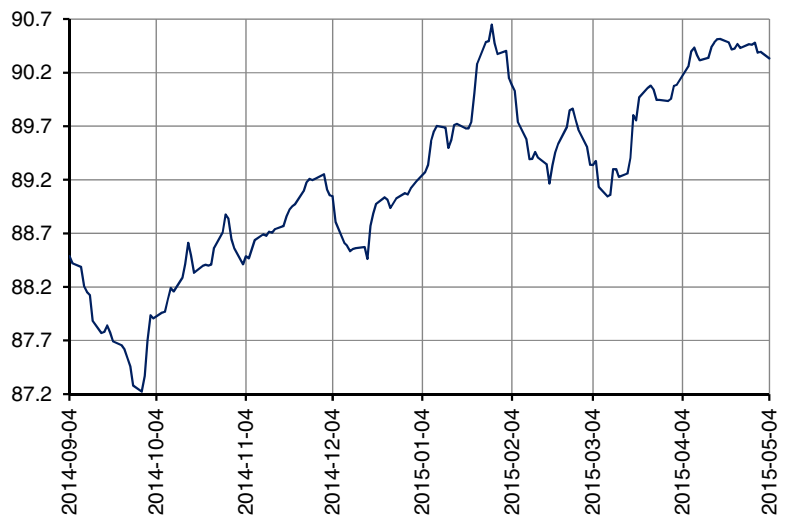

Figura 11. Valuación del bono.

Fuente: elaboración propia a través de hoja de cálculo.

presentan un mínimo de $\$ 0.00$ y un máximo de $\$ 395.1987$, con un diferencial de $\$ 395.1987$. La diferencia mínima entre las valuaciones de los modelos utilizados durante el periodo de valuación fue de $\$ 154.5342$ el 16 de octubre de 2014, y la diferencia máxima fue de $\$ 402.6041$ el 17 de abril de 2015. Se puede observar que las valuaciones log-gaussianas son mayores que las valuaciones log-estables, confirmando que el modelo log-gaussiano sobrestima los eventos de bajo impacto financiero y económico. Las valuaciones log-estables se aproximan a las valuaciones log-gaussianas cuando el Eurostoxx incrementa o desciende y los otros factores permanecen constantes. La diferencia máxima es de $\$ 482.9548$ y se presenta cuando el índice alcanza los 4,180 puntos; al incrementar o disminuir la diferencia es decreciente. La valuación del bono se presenta en la figura 11, donde se muestran las valuaciones del bono en función de la estructura de las tasas de interés cupón cero. Las valuaciones presentan un mínimo de $\$ 87.2236$ y un máximo de $\$ 90.6480$, con un diferencial de $\$ 3.4244$. En el prospecto del bono bancario estructurado referenciado al índice SX5E se indica que el pago, en pesos, es el monto siguiente:

$$
c\left(t, M_{t}, B_{t}\right)=B_{t}+100 F_{0} \max _{M_{t}}\left(\frac{c\left(t, M_{t}\right)}{S}, 0\right)
$$

donde el producto de cien veces el factor de participación y el valor máximo del cociente entre la valuación de la opción sobre el Eurostoxx y el precio de liquidación o cero es la monetización de las opciones y representa las ganancias adicionales, en pesos, que los inversionistas pueden obtener con el producto estructurado. En la fecha de emisión el valor del bono es $\$ 88.4874$, y el capital restante para la posición larga en opciones europeas de compra sobre el Eurostoxx es de $\$ 11.5126$, por lo que el factor de participación para el modelo log-gaussiano es de solo una opción, porque el precio de la opción europea es $\$ 328.9045$, la monetización es \$10.3669 y el emisor obtiene una comisión de $\$ 1.1457$ por cada producto estructurado para un precio de emisión de \$100.00; el factor de participación para el modelo log-estable es de quince opciones, debido a que el precio de las opciones asciende a $\$ 20.9925$, la monetización es $\$ 0.6617$ y el emisor obtiene una comisión de $\$ 1.5875$ por cada producto estructurado para un precio de emisión de $\$ 100.00$. Las valuaciones de los productos estructurados a través del modelo log-gaussiano y log-estable se presentan en la figura 12, donde se muestran las valuaciones del producto estructurado de capital garantizado a través modelo log-gaussiano (línea cielo), las valuaciones del producto estructurado a través del modelo log-estable (línea marino) y los precios de mercado (línea punteada roja), que 


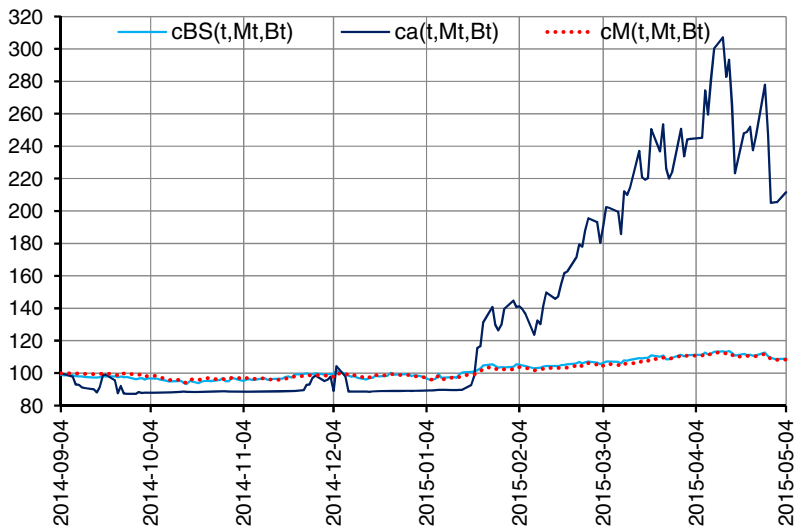

Figura 12. Valuación del producto estructurado de capital garantizado.

Fuente: elaboración propia a través de hoja de cálculo.

son semejantes a las valuaciones log-gaussianas. Las valuaciones log-gaussianas presentan un mínimo de \$93.3655 y un máximo de $\$ 113.7071$, con un diferencial de $\$ 20.3416$; las valuaciones log-estables presentan un mínimo de $\$ 87.2237$ y un máximo de $\$ 277.1856$, con un diferencial de $\$ 189.9619$, y los precios de mercado presentan un mínimo de $\$ 94.1255$, un máximo de $\$ 112.8350$ y un diferencial de $\$ 18.7095$. Las valuaciones log-gaussianas son mayores que las valuaciones log-estables hasta el 20 de enero de 2015, y a partir del 21 de enero de 2015 las valuaciones logestables son superiores a las log-gaussianas, confirmando que el modelo log-gaussiano sobrestima eventos de bajo impacto financiero y económico y subestima eventos de alto impacto financiero y económico. Las valuaciones log-estables cuantifican más adecuadamente el riesgo de mercado y las ganancias se reflejan cuando el valor del índice incrementa y la valuación de las opciones log-estables también incrementa, y además el factor de participación multiplica las ganancias de la nota estructurada. El modelo log-estable permite mayores comisiones para los emisores por cada producto estructurado y permite obtener mayores ganancias a los inversionistas. Las valuaciones de los productos estructurados del modelo log-gaussiano y el precio de mercado se presentan en la figura 13, en la que se muestran los valores producto estructurado log-gaussiano

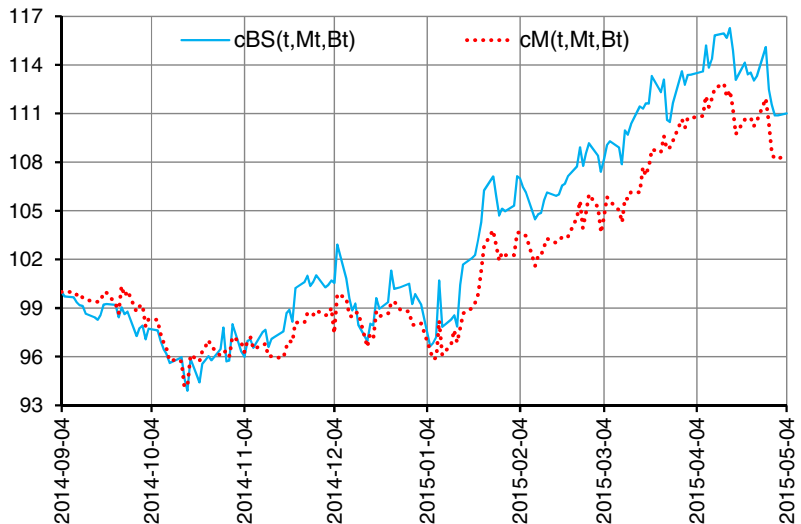

Figura 13. Valuación del producto estructurado y los precios de mercado. Fuente: elaboración propia a través de hoja de cálculo. 


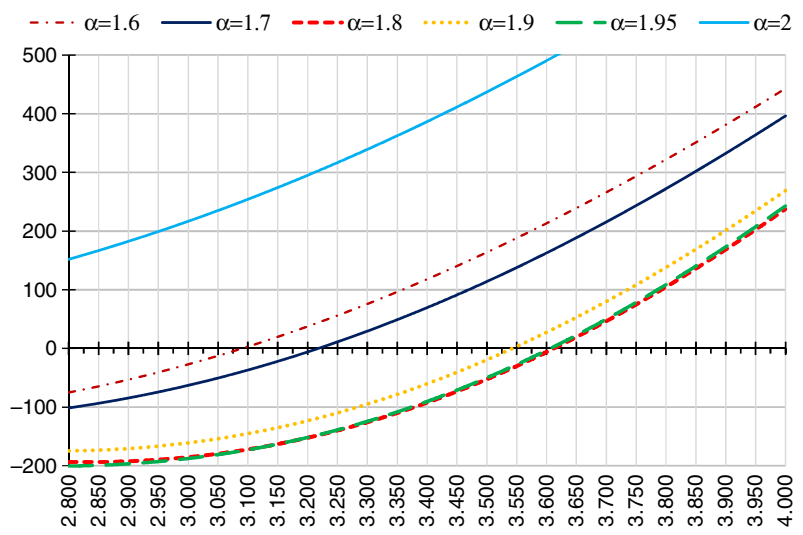

Figura 14. Precios de las opciones en función del subyacente y del parámetro de estabilidad. Fuente: elaboración propia a través de hoja de cálculo.

(línea cielo) y los precios de mercado (línea punteada roja); la diferencia máxima del producto estructurado log-gaussiano y el precio de mercado es $\$ 3.1012$, que se presentó el 16 de marzo de 2015 , representando una diferencia del $2.9221 \%$, y la mínima es $\$ 0.0089$, que se presentó el 18 de diciembre de 2014, representando una diferencia del $0.0089 \%$. Se puede observar que en las fechas más recientes el valor de mercado es menor que el precio teórico del modelo loggaussiano, lo que disminuye las ganancias de los inversionistas. Los precios de las opciones en función del subyacente y del parámetro de estabilidad se presentan en la figura 14, en la que se pueden apreciar los precios de la opción log-gaussiana (línea cielo) y los precios de las opciones log-estables en función del valor de subyacente (incluye el mínimo 2,874 puntos y el máximo 3,828 puntos durante el periodo de valuación), del parámetro de estabilidad (línea marino 1.6945, línea discontinua y punteada púrpura 1.6, línea discontinua roja 1.8, línea punteada amarilla 1.9 y línea discontinua verde 1.95) y sin considerar el límite inferior en el valor de las opciones; en la fecha de valuación, el índice presentó un valor de 3,277.25 puntos y el valor teórico de la opción log-gaussiana y los valores teóricos de las opciones log-estables, que se encontraban dentro de dinero, fueron positivos, \$328.9045 y \$20.9925, respectivamente; se puede apreciar que la valuación de la opción log-gaussiana sobrestima las valuaciones de las opciones log-estables con diferentes parámetros de estabilidad y el factor de participación del modelo log-estable es superior al factor del modelo log-gaussiano, multiplicando las ganancias de los inversionistas log-estables quince veces para la distribución estimada $f_{X}(x, 1.6945,-0.1707)$ cuando el índice rebasa los 3,245 puntos el día 21 de enero de 2015, por lo que el factor de participación es el multiplicador que permite a los inversionistas log-estables obtener mayores ganancias cuando el índice aumenta su valor, ya que multiplica las ganancias por el pago de las opciones dentro de dinero y, en caso de que las opciones fuera de dinero no obtengan ganancias, entonces los inversionistas solo reciben el valor nominal del bono en la fecha de vencimiento; también se puede observar que los precios log-estables son inferiores a los log-gaussianos en una posición cercana a las opciones en dinero. Este comportamiento es explicado porque la función de densidad log-gaussiana presenta una masa de probabilidad mayor en valores adyacentes al precio de liquidación y las funciones de densidad log-estables presentan una masa de probabilidad en los extremos de la distribución; la mayoría de las opciones son valuadas con precios de liquidación adyacentes al precio forward o precio futuro del subyacente como un precio justo considerando los insumos del mercado en la fecha de negociación, por lo que las opciones son valuadas en un intervalo adyacente a cuando 
están en dinero, un poco fuera de dinero o un poco dentro de dinero, pero no demasiado fuera de dinero o demasiado dentro de dinero, que es cuando las opciones log-estables incrementan su valor, pudiendo tener factores de participación menores que las opciones log-gaussianas.

El precio de mercado del producto estructurado de capital garantizado es similar al precio teórico; sin embargo, el precio de mercado no está disponible al público en general, por lo que se han descrito las características del producto. Se utilizan los insumos del mercado para la valuación, innovando al modelar la curtosis y la asimetría a través de las distribuciones logestables que ninguno de los trabajos citados ha realizado, porque han utilizado la distribución log-gaussiana. El atractivo de la nota estructurada de capital garantizado es que el diagrama de pago indica que el rendimiento esperado por los inversionistas puede ser teóricamente ilimitado, por lo que se calcula un intervalo de confianza para el Eurostoxx a través de:

$$
\wp\left(-\gamma \tau^{\frac{1}{\alpha}} z_{\frac{\zeta}{2}} \leq \ln \left(M_{T}\right)-\ln \left(M_{0}\right)+\left(i-r-\beta \gamma^{\alpha} \sec (\theta)\right) \tau \leq \gamma \tau^{\frac{1}{\alpha}} z_{\frac{\zeta}{2}}\right)=1-\zeta
$$

El precio subyacente en la fecha de emisión es $M_{0}=3,277.25$, el parámetro de escala histórico anual es $\gamma=0.25470617$ y la distribución de los rendimientos del Eurostoxx es $Z \sim S_{1}$ (1.6945, -0.1707); entonces, a través de la ecuación (12) se calcula el intervalo de confianza del $95 \%$ para los 170 días hábiles donde se ha valuado el producto estructurado, siendo este $1,556.93 \leq M_{T} \leq 6,297.03$, que incluye al intervalo de confianza cuando $Z \sim S_{1}$ (2), que es $2,318.08 \leq M_{T} \leq 4,662.78$. Los valores del índice previos a la fecha de valuación son 1,995.01 como mínimo y 3,314.78 como máximo, donde el valor mínimo está fuera del intervalo loggaussiano y los valores del índice durante el periodo de valuación son 2,874.65 como mínimo y 3,828.78 como máximo, periodo en el que no se observaron eventos de alto impacto financiero y económico. El intervalo de confianza indica que el valor del índice tiende a bajar con mayor frecuencia que a subir, información que los inversionistas desconocen. El intervalo de confianza del $95 \%$ durante el periodo de vigencia del producto estructurado para el precio subyacente en la fecha de vencimiento es $1,556.93 \leq M_{T} \leq 6,297.03$, que incluye el intervalo de confianza cuando $Z \sim S_{1}$ (2), que es $2,169.73 \leq M_{T} \leq 4,995.23$. La probabilidad de obtener ganancias en la fecha de vencimiento se calcula mediante:

$$
P\left(M_{T}>S\right)=\int_{Z}^{\infty} f_{Z_{\tau}^{\mathcal{Q}}}\left(z_{\tau}^{\mathcal{Q}} ; \alpha,-\beta\right) d Z_{\tau}^{\mathcal{Q}}=\int_{Z}^{\infty} f_{Z_{\tau}^{\mathcal{Q}}}\left(-z_{\tau}^{\mathcal{Q}} ; \alpha, \beta\right) d Z_{\tau}^{\mathcal{Q}}
$$

donde:

$$
Z=\frac{\ln (S)-\ln \left(M_{t}\right)+\left(i-r-\beta \gamma^{\alpha} \sec (\theta)\right) \tau}{\gamma \tau^{\frac{1}{\alpha}}}
$$

Las probabilidades en función del valor del índice se presentan en la figura 15, y también las probabilidades de que la variable $Z$ (valor del índice) supere el valor indicado en el eje horizontal en la fecha de vencimiento - como se puede apreciar las probabilidades log-gaussianas (línea cielo) — son superiores a las probabilidades log-estables (línea marino) hasta que valor del índice supera los 3,390 puntos. Ambas probabilidades son decrecientes, esto es, las probabilidades de obtener ganancias mayores son monótonas decrecientes; por ejemplo, la probabilidad de que las opciones log-estables se encuentren dentro de dinero es del $47.69 \%$, y la probabilidad de que las opciones log-gaussianas se encuentren dentro de dinero es del $49.60 \%$ cuando el valor del índice supera los 3,172.63 puntos, mientras que las probabilidades de que el valor del índice supere los 4,000 puntos son del 34.45 y del $30.03 \%$ para las opciones log-estables y log-gaussianas, respectivamente. El logaritmo de la frecuencia del rendimiento subyacente 


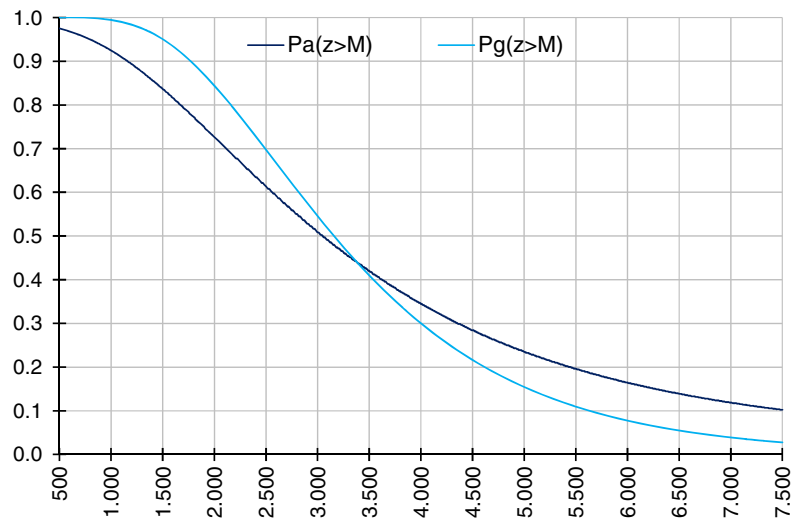

Figura 15. Probabilidades en función del valor del índice. Fuente: elaboración propia a través de hoja de cálculo.

se presenta en la figura 16, en la que se puede apreciar que la estimación de los rendimientos es más adecuada a través de la distribución log-estable (línea marino), ya que modela más adecuadamente la curtosis y la asimetría de los rendimientos observados (puntos negros) que la distribución log-gaussiana (línea cielo), por lo que el modelo log-estable cuantifica mejor que el modelo log-gaussiano las frecuencias de pérdidas y ganancias de alto y bajo impacto financiero y económico por la inversión en el Eurostoxx, las opciones sobre el Eurostoxx y los productos estructurados de capital garantizado valuados en este trabajo.

Los mercados financieros no son completos porque la cobertura perfecta no existe, y en los mercados incompletos no es posible transferir los riesgos completamente. Suponiendo que los mercados son completos, se tiene una visión irreal de la medida neutral al riesgo al valuar opciones. Los mercados incompletos son más eficientes porque las coberturas de riesgos se pueden cuantificar para minimizar los riesgos; la falta de completitud de los mercados financieros se presenta por los niveles de comercialización en relación con los riesgos que se necesitan cubrir, por el desconocimiento del modelo apropiado para modelar los rendimientos y principalmente por

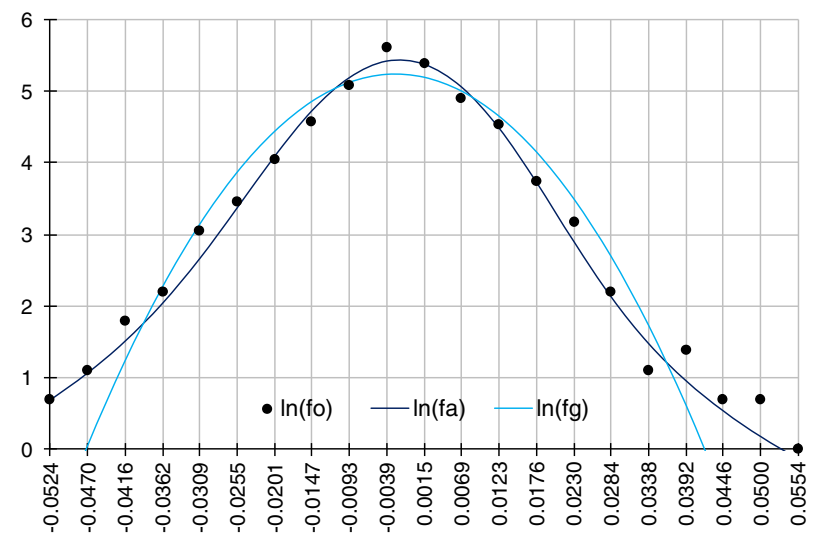

Figura 16. Logaritmo de las frecuencias de los rendimientos subyacentes. Fuente: elaboración propia a través de hoja de cálculo. 
las discontinuidades de los precios. Las martingalas equivalentes de los mercados incompletos permiten valuar opciones de formas distintas, y estas probabilidades se deben interpretar como el valor que los inversionistas apuestan en el evento. El parámetro de estabilidad proporciona información acerca del comportamiento del proceso: cuando $\alpha$ se aproxima a dos, el proceso presenta gran cantidad de oscilaciones de bajo impacto financiero (rendimientos cercanos a cero) entre los saltos de alto impacto financiero (rendimientos que generan pérdidas o ganancias moderadas), y cuando $\alpha$ se aproxima a la unidad (proceso de Lévy) los precios de las opciones cambian debido a los saltos que generan pérdidas o ganancias elevadas y la presencia de periodos de estabilidad entre los saltos, como se puede observar en las figuras 1-6 y que son capturados más adecuadamente por los procesos log-estables, ya que capturan las oscilaciones de bajo impacto financiero a través del proceso de Wiener y los saltos de alto impacto financiero que generan pérdidas o ganancias a través de los procesos de Poisson. Los procesos log-estables modelan más adecuadamente los rendimientos que el proceso log-gaussiano, porque el proceso log-gaussiano es un caso particular (simétrico) de los procesos log-estables que capturan la curtosis y la asimetría de los rendimientos de los mercados, como se puede observar en las figuras 4, 5, 15 y 16, además de que satisfacen las propiedades de que el valor de las opciones con pagos positivos (dentro de dinero) debe ser positivo, por lo que las opciones de compra con pagos nulos (fuera de dinero) deben ser menos valiosas que las opciones de compra dentro de dinero, como se puede observar en la figura 14, pudiendo tener valores negativos que darían oportunidades de arbitraje. Las pruebas cualitativas que muestran que los procesos log-estables superan al proceso log-gaussiano se pueden observar en la figura 16, y las pruebas cuantitativas están confirmadas en las tablas 2-4, por lo que la valuación de los reclamos contingentes log-estables cuantifican más adecuadamente el riesgo de mercado (riesgo de precio), que es una característica importante en las matemáticas financieras para que los precios de las opciones sean más justos para los emisores y los inversionistas (poseedores), lo que permite a la ingeniería financiera ser más eficiente en la valuación, proporcionando a los emisores e inversionistas herramientas más adecuadas y que se apegan con mayor medida a la realidad para generar instrumentos de inversión que se adaptan a las necesidades y a la realidad de los mercados.

\section{Conclusiones}

Las valuaciones de las opciones log-gaussianas son mayores que las valuaciones log-estables, confirmando que el modelo log-gaussiano sobrestima los eventos de bajo impacto financiero utilizando los mismos factores endógenos y exógenos disponibles. Debido a que las valuaciones log-estables se aproximan a las valuaciones log-gaussianas cuando el Eurostoxx incrementa o desciende, mientras los insumos permanecen constantes, esto es un indicador de que los pagos contingentes log-estables están mejor cuantificados que los pagos contingentes log-gaussianos, porque estos últimos subestiman los eventos de alto impacto financiero que se traducen en ganancias o pérdidas significativas que no están consideradas adecuadamente por los seguros log-gaussianos, y están mejor modelados por los seguros log-estables y están sustentados cuantitativamente por los estadísticos de bondad de ajuste.

Las comparaciones de los productos estructurados log-estables y log-gaussianos muestran que las valuaciones log-gaussianas son mayores que las valuaciones log-estables mientras el valor del índice es menor que 3,269.18 puntos, y cuando el valor del índice supera los 3,269.18 puntos, las valuaciones log-estables son superiores que las valuaciones log-gaussianas, confirmando la sobrestimación de eventos de bajo impacto financiero cuando el índice está cercano al precio de liquidación y la subestimación de eventos de alto impacto financiero que generan mayores ganancias para los inversionistas log-estables porque cuantifican más adecuadamente el riesgo de 
precio. Las ganancias se presentan cuando el índice supera los 3,269.18 puntos y las valuaciones log-estables incrementan con respecto a las log-gaussianas. El factor de participación log-estable potencia las ganancias porque incluye quince opciones que multiplican el pago en la fecha de liquidación, mientras que el factor de participación log-gaussiano incluye una única opción para el mismo pago. El modelo log-estable otorga mayores comisiones por cada producto estructurado a los emisores y otorga mayores ganancias a los inversionistas debido a la diferencia en el factor de participación y que depende de la valuación adecuada de las opciones en el momento de negociación.

La valuación de productos estructurados depende de la correcta cuantificación del riesgo de precio y los procesos log-estables han mostrado que superan al proceso log-gaussiano modelando adecuadamente la curtosis y la asimetría, permitiendo que la valuación de las opciones se realice de forma más apegada a la realidad con los insumos disponibles. La estimación de la distribución de los rendimientos y la validación cuantitativa permiten observar que el proceso log-gaussiano sobrestima los eventos que no generan pérdidas o ganancias significativas, y subestima los eventos que generan pérdidas o ganancias significativas y que se encuentran en los extremos de la distribución. A través del parámetro de estabilidad, los procesos log-estables capturan más adecuadamente los eventos lejanos a la moda para valuar más adecuadamente las pérdidas o ganancias significativas, y a través del parámetro de asimetría las opciones dentro de dinero presentan valuaciones superiores a las opciones fuera de dinero.

En investigaciones futuras se pueden valuar otro tipo de productos estructurados sobre contratos a plazo (forwards), futuros, swaps u opciones sobre otros subyacentes con otras características y creando otro tipo de cobertura a través de distribuciones log-estables.

\section{Referencias}

Aguilar-Juárez, I.P. y F. Venegas-Martínez (2014). Una estrategia de inversión y cobertura mediante la combinación de notas estructuradas, Working Paper, Munich Personal RePEc Archive [consultado 10 Dic 2015]. Disponible en: http://mpra.ub.uni-muenchen.de/558928/.

Blümke, A. (2009). How to Invest in Structured Products: A Guide for Investors and Asset Managers. John Wiley \& Sons. http://dx.doi.org/10.1002/9781119206590

Cao, J. y Rieger, M. O. (2013). Risk classes for structured products: mathematical aspects and their implications on behavioral investors. Annals of Finance, 9(2), 167-183. http://dx.doi.org/10.2139/ssrn.2065216

Castillo Miranda, H. (2008). Notas Estructuradas. Instituto Mexicano de Ejecutivos de Finanzas, IMEF.

Čížek, P., Härdle, W. y Weron, R. (2005). Stable Distributions. Statistical Tools for Finance and Insurance. pp. 21-44. Berlin: Springer.

Climent-Hernández, J. A. y Venegas-Martínez, F. (2013). Valuación de opciones sobre subyacentes con rendimientos $\alpha-$ estables. Revista de Contaduría y Administración, 58(4), 119-150. http://dx.doi.org/10.1016/S0186-1042(13)71236-1

Contreras Piedragil, C. E. y Venegas-Martínez, F. (2011). Valuación de opciones sobre activos subyacentes con distribuciones estables. Estocástica: Finanzas y Riesgo, 1(1), 55-71.

Dostoglou, S. y Rachev, S. T. (1999). Stable distributions and term structure of interest rates. Mathematical and Computer Modelling, 29(10), 57-60. http://dx.doi.org/10.1016/S0895-7177(99)00092-8

Gómez Almaraz, G. (2007). Los beneficios de incorporar los productos estructurados en los portafolios tradicionales de inversión. Universidad Nacional Autónoma de México.

Hens, T. y Rieger, M. O. (2014). Can utility optimization explain the demand for structured investment products? Quantitative Finance, 14(4), 673-681. http://dx.doi.org/10.1080/14697688.2013.823512

Ilin, I. V., Koposov, V. I. y Levina, A. I. (2014). Model of asset portfolio improvement in structured investment products. Life Science Journal, 11(11), 265-269.

Lamothe Fernández, P. y Pérez Somalo, M. (2003). Opciones Financieras y Productos Estructurados (2. ${ }^{a}$ edición). España: McGraw Hill.

Mascareñas, J. (2010). Productos Financieros Estructurados, Monografías de Juan Mascareñas sobre Finanzas Corporativas, Universidad Complutense de Madrid. 
McCann, K. y Cilia, J. (1994). Structured Notes. Federal Reserve Bank of Chicago. Financial Markets Unit.

Nolan, J. P. (2001). Maximum likelihood estimation and diagnostics for stable distributions. En O. E. BarndorffNielsen, T. Mikosch, y S. Resnick (Eds.), Lévy Processes (pp. 379-400). Boston: Brikhäuser. http://dx.doi.org/ 10.1007/978-1-4612-0197-7_17

Ortiz-Ramírez, A., Venegas-Martínez, F. y López-Herrera, F. (2011). Valuación de una nota estructurada que liga el rendimiento de un índice bursátil con los pagos de un bono y un derivado. Estocástica: Finanzas y Riesgo, 1(2), 49-62.

Scalas, E. y K. Kim (2006). The Art of Fitting Financial Time Series with Levy Stable Distributions, Munich Personal RePEc Archive August (336): 1-17 [consultado 8 Feb 2012]. Disponible en: mpra.ub.uni-muenchen.de/336.

Schroff, S., S. Meyer y H. P. Burghof (2015): Retail investor information demand — speculating and investing in structured products, The European Journal of Finance [consultado 4 May 2015]. Disponible en: http://dx.doi.org/10.1080/1351847X.2015.1020392: 1-23.

Venegas-Martínez, F. (2008). Riesgos financieros y económicos: productos derivados y decisiones económicas bajo incertidumbre (2. ${ }^{\mathrm{a}}$ edición). Cengage Learning Latin America.

Wallmeier, M. (2011). Beyond payoff diagrams: How to present risk and return characteristics of structured products. Financial Markets and Portfolio Management, 25(3), 313-338. http://dx.doi.org/10.2139/ssrn.1694162 